\title{
EL LUGAR DE LA PSICOLOGÍA EN LA EPISTEMOLOGÍA DE KUHN: LA POSIBILIDAD DE UNA PSICOLOGÍA DE LA INVESTIGACIÓN CIENTÍFICA
}

\section{THE PLACE OF PSYCHOLOGY IN KUHN'S EPISTEMOLOGY: THE POSSIBILITY OF A SCIENTIFIC RESEARCH PSYCHOLOGY}

Dr. Juan Brunetti (juanbrunetti@gmail.com) y Dra. Elizabeth Beatriz Ormart (eormart@psi.uba.ar). Facultad de Psicología, Universidad de Buenos Aires (Buenos Aires, Argentina).

\section{Abstract}

Kuhn's epistemology has inspired research within psychology of knowledge. At the same time, researchers have taken Kuhn's categories to theorize on their findings. Finally, Kuhn himself turned towards them to illuminate his concept of scientific development and to build the foundation of a scientific research psychology. The issue about psychology epistemological rules is at the background of these reciprocal influences.

Keywords: epistemology, psychology, science, learning, knowledge.

\section{Resumen}

La epistemología de Kuhn ha inspirado investigaciones en el ámbito de la psicología del conocimiento. Del mismo modo los psicólogos investigadores de los procesos psico-cognitivos han tomado categorías kuhnianas para teorizar sus hallazgos. Finalmente, el mismo Kuhn se volvió hacia ellos para iluminar su concepción del desarrollo científico y construir los cimientos de una psicología de la investigación científica. Por otra parte, la cuestión acerca del estatuto epistemológico de la psicología está en la base de estas influencias recíprocas.

Palabras clave: epistemología, psicología, ciencia, aprendizaje, conocimiento.

\section{Introducción}

El presente escrito (1) busca, en primer lugar, describir la atención que han merecido en la epistemología de Kuhn las cuestiones psicológicas del conocimiento. En su recorrido epistemológico, Kuhn transita por los más diversos modelos explicativos de la psicología. A lo largo de la obra de Kuhn encontramos referencias a la Gestalt, al asociacionismo, a Piaget y a la psicología cognitiva, intrincadas en un fértil diálogo con la generación del conocimiento científico. En el presente artículo se aborda de un modo general el interés manifestado en Kuhn por el funcionamiento psíquico cognoscitivo en los procesos de producción de 
conocimiento científico.

En segundo término, nos abocamos a interrogar desde el modelo kuhniano el estatuto científico de la psicología. En este caso, los intereses que nos guiaron apuntan al valor científico de las teorías psicológicas en Kuhn. En otras palabras, la cuestión a discutir es si en la obra de Kuhn a la psicología le es otorgado el estatuto de ciencia o si ella se encuentra aún en un proceso pre-científico. Y aún en este último caso, habría que revisar el sentido dado por Kuhn a la etapa anterior a la ciencia normal, de acuerdo con las exigencias o parámetros de cientificidad asumidos por el autor.

\section{1. ¿Cómo relacionó tempranamente Kuhn procesos cognitivos y conocimiento científico?}

Desde muy temprano Kuhn ha querido saber qué es lo que pasa con los científicos particulares. En las Conferencias Lowell (2) señalaba que, en el estudio del método, hay que incorporar el estudio del agente de la teoría, entiéndase por tal tanto al individuo como la comunidad (Mayoral de Lucas 2004:198). Allí planteaba que el lenguaje es lo que conecta al individuo con el grupo a través de la teoría. Una teoría es un lenguaje compartido por numerosos individuos, incluso a lo largo de mucho tiempo. Por lo tanto, por más que se objetive, la ciencia surge de las condiciones epistemológicas de los sujetos. Según Mayoral de Lucas: "A lo largo de su etapa como fellow, Kuhn explorará las condiciones epistemológicas del científico desde diversos puntos de vista (lógico-semántico y psicológico, principalmente)" (2004:199).

En su primera etapa, Kuhn ha atendido a las cuestiones cognitivas dándoles un lugar de preeminencia. En La Revolución Copernicana (1957) intuía que en un cambio de esquema conceptual debía haber más que problemas estrictamente científicos y se preguntaba por el motivo que lleva a los sujetos a cambiar un esquema exitoso por otro. Las razones pueden ser epistemológicas e inciden en ellas variables históricas. Sin embargo, comprender mejor esos cambios implicaría descubrir cómo es que son psico-cognitivamente posibles. En La Estructura (1962) volvía una y otra vez a reflexionar sobre cómo los científicos concretos vieron y resolvieron sus problemas y dieron lugar a nuevos modos de entender la ciencia gestando cambios revolucionarios (Kuhn 1962/2002a: 99, 144, 210, 238). Por otra parte, debemos convenir en que si una comunidad cambia sus convicciones paradigmáticas, es porque se han ejecutado para ello operaciones psicológicas en sus miembros. Por presión de grupo o sin ella el individuo pone en juego factores cognitivos que el cambio exige. Sabemos que los miembros de una comunidad científica, en el período de revolución, se van "convirtiendo" primeramente de a uno y hasta en algunos casos podríamos ubicar al que comienza la serie. ¿Cómo es que la ciencia cambió su punto de vista de un objeto balanceándose de una cuerda, de modo que pasara de ser un cuerpo que se desplaza de una posición superior a una más baja hasta llegar a su reposo natural con dificultad, a ser un péndulo? Según Kuhn: “¿Por qué tuvo lugar ese cambio de visión? ¿Por el genio individual de Galileo?" (1962/2002a:188).

También es cierto que Kuhn ha propuesto un cambio en masa de la comunidad en donde la mayoría podría convertirse en un golpe de mando revolucionario. Por supuesto que al historiador de la ciencia le interesa destacar esos momentos en que ciertas teorías adquieren confiabilidad y son validadas públicamente por parte de la mayoría de los científicos. Pero cacaso ese proceso masivo elimina de los individuos la exigencia operacional a nivel psicológico en sus mentes, el compromiso con un grado de racionalidad y la necesidad de comprensión? Probablemente esos procesos inmanentes en los que unos empiezan a aceptar el punto de vista de otros sea el mayor aporte de racionalidad que el individuo realiza en los cambios revolucionarios.

A pesar de que Kuhn haya manifestado su interés en la psicología social, no ha dejado de suministrar 
ejemplos acerca del funcionamiento cognitivo del sujeto científico particular y, podría decirse, que una de sus preocupaciones ha sido dar cuenta de lo que sucede en la mente del científico, tanto en el período de ciencia normal como en los procesos revolucionarios. El Kuhn historiador no se ha limitado al relato de los hechos. Su estilo está lleno de insinuaciones respecto de lo que podría suceder en el interior del científico, como si pretendiera leer sus mentes: "Yo pensaba -perdónenme- que, con la posible excepción de Koyré, y quizá incluso ni eso, era capaz de leer textos y meterme en la cabeza de las personas que los escribieron mejor que ningún otro en el mundo" (Baltas, Gavroglu y Kindi 2002:322).

Lo que pretendemos destacar aquí es que la investigación de Kuhn acerca de la historia de la ciencia lo ha conducido inevitablemente hacia el terreno de la psicología del conocimiento del investigador.

\section{El enfoque reconstructivo de Kuhn}

El enfoque de Kuhn podría ser denominado reconstructivo, es decir, partiendo de los productos de la ciencia se dirige hacia las comunidades; de modo que no sorprende que lo primero en advertir sean las realizaciones científicas y el segundo paso sea atribuírselas a lo que acontece en una comunidad de científicos. Pero avanzando un poco más, no queda sino reconocer que dentro de la comunidad existen individuos reales. A partir de ahí se puede avanzar sobre la naturaleza de esos procesos en la mente del hombre de ciencia particular y concreto que posee funciones psicológicas determinadas. Luego, desde ahí, los momentos de ciencia normal y los acontecimientos revolucionarios adquieren nueva claridad. Explicar en qué consisten esos procesos no ha sido una tarea sencilla para Kuhn, el intento de descubrirlos lo ha llevado por diversos caminos de la psicología relacionada con las capacidades cognitivas. Reconocer esto implica encarar una exploración en la obra de Kuhn. A este respecto se hace imprescindible destacar que el pensamiento epistemológico de Kuhn manifiesta transformaciones y esta cuestión es ya un capítulo aparte. Si tomamos como referencia el Kuhn de La Estructura (1962) encontramos una serie de afirmaciones que parecen atenuarse en su radicalidad en el último Kuhn (década del '90). En medio de ambos períodos podemos ubicar una etapa de transición en la que nuestro autor intenta responder a sus críticos y replantearse sus primeras ideas. Para esta investigación toma relevancia cómo Kuhn emplea, en cada uno de estos momentos, los referentes psicológicos de procesos cognitivos para aclarar sus puntos de vista. Por lo dicho la exploración acerca del uso de la psicología del conocimiento en Kuhn no sólo es posible, sino altamente relevante para comprender su concepción epistemológica.

\section{3. ¿Cuál es el grado de interés de Kuhn por el funcionamiento psíquico cognoscitivo?}

El interés de Kuhn en las cuestiones cognitivas viene de manera secundaria a complementar lo que en él es su búsqueda primaria, es decir, su interés en la construcción y desarrollo del conocimiento científico. Pero no hay que explicar demasiado para comprender que la ciencia es un producto del conocimiento del hombre, la expresión epistemología así lo revela. Cómo es que en la ciencia intervienen otros factores: sociológicos, históricos, económicos, culturales, etc., lo cual no significa que haya que perder de vista que la ciencia es un tipo de conocimiento. Por lo tanto, aunque no sea la única variable, tampoco es un tema menor en cualquier epistemología considerar una teoría que sirva de referencia de los procesos de conocimiento que ocurren en un psiquismo o, dicho en forma más específica, en un sujeto epistémico. Aquí es donde las utilizaciones de Kuhn de teorías psicológicas se vuelven relevantes con una proyección epistemológica que deberíamos realzar. 


\subsection{Los cambios científicos como cambios cognitivos}

En su epistemología Thomas Kuhn ha incluido aspectos de la psicología de los procesos cognitivos. La relación que guardan la epistemología y la psicología del conocimiento es bidireccional, ya que ambas se encuentran mutuamente referidas. En este sentido, podemos mencionar algunos ejemplos:

a) Kuhn ha sostenido, en varias oportunidades, que los datos que aportan los psicólogos pueden iluminar la epistemología: "si esta suerte de estrategia es razonablemente legítima, constituye un error pedir a la persona que la usa que trace fronteras y diga cuándo es psicología, cuándo historia, y cuándo filosofía de la ciencia. Evidentemente puede ser cualquiera de estas cosas sin las otras. Pero esto no implica que los datos del psicólogo o las generalizaciones del psicólogo, justamente por ser del psicólogo, no puedan iluminar a quien tenga intereses epistemológicos" (Kuhn 1974/1978:83).

b) Asimismo, ha sido muy explícito, en referencia con las influencias que ha recibido a lo largo de su obra. Kuhn señala que la historia de la ciencia y la psicología de Piaget han influido recíprocamente en su pensamiento (Kuhn 1971/1996:46).

c) Finalmente, cabe destacar los acercamientos de Kuhn a trabajos de psicólogos cognitivos como Carey (2004) o Rosch (1999). Este interés de Kuhn por la psicología del conocimiento no ha pretendido sino alumbrar lo que él mismo comprobaba desde una epistemología que atiende a la realidad histórica del acaecer científico.

Que los trabajos de Kuhn fueron hechos dentro de un eje claramente epistemológico no es una cuestión sometida a disputa. Como epistemólogo su mirada ha estado enfocada a esa construcción humana, grupal e histórica que es la ciencia. Podría entenderse, entonces, que la psicología ha jugado un papel menor, meramente ilustrativo o hasta irrelevante en su obra. Todas estas suposiciones parecen desconocer ciertos datos que se encuentran presentes a lo largo de la obra de Kuhn, algunos de los cuales hemos mencionado anteriormente. Lo cierto, es que su modo de pensar la ciencia ha incluido la historia y la psicología del conocimiento en una trabazón substancial.

Es difícil leer la obra de Kuhn en detalle, incluyendo sus declaraciones autobiográficas y los datos de sus últimos intereses, sin otorgar que la psicología del conocimiento haya formado parte de su búsqueda epistemológica, claramente identificada, por lo demás, dentro del cuadro de la epistemología naturalizada.

No obstante es verdad que aun otorgando que Kuhn viera a la psicología como necesaria para complementar su lectura de los procesos científicos, queda por responder el problema básico de esta relación. ¿Qué modelo de explicación psicológica le resulta fecundo para comprender los procesos de construcción y cambio científico? ¿Estos procesos son individuales o colectivos?

La ciencia, en tanto que es una construcción social, sucede en las comunidades y los recursos psicológicos a los que acude Kuhn están relacionados con la psicología de los individuos.

En principio, no podía esto ser de otra manera ya que la psicología del conocimiento se dirige hacia los psiquismos individuales. Discutir este punto sería plantear la validez de utilizar la psicología del conocimiento para la epistemología. En otras palabras, sería cuestionar la legitimidad de cierta línea de la epistemología naturalizada. Pero sin salirnos del propio Kuhn se puede decir que él observa esta dificultad. Así es como acepta que las comunidades, en tanto colectivo, no tienen conocimientos y la referencia a los 
cambios de Gestalt que ellas sufrirían, por ejemplo, no debe entenderse al pie de la letra. Las comunidades no piensan pero los sujetos que la componen sí. La teoría de la Gestalt colaboró con alumbrar suficientemente los procesos psicológicos implicados en la investigación científica. Le resultó útil a Kuhn para comprender la inconmensurabilidad de los paradigmas desde la mirada del historiador, pero le resultó insuficiente para dar cuenta de los procesos psicológicos involucrados en ese cambio.

Cuando Kuhn percibió estas dificultades se despegó de la Gestalt y comenzó a buscar en otras explicaciones psicológicas la clave de los cambios científicos. Así, pudo ver que en el cambio científico es posible incluir elementos de un núcleo anterior con otros nuevos producidos por ese mismo núcleo como continuidades que permanecen después del cambio (Kuhn 1976/2002b:218). Esto último puede ser entendido mejor desde la psicología genética de Piaget o la psicología cognitiva, en particular en las investigaciones de Carey respecto del desarrollo en los niños (Nersessian 2003:205, nota 2 al pie). Sobre esto mismo cabe recordar que, en sus comienzos, Kuhn buscó encontrar en Piaget explicaciones psicológicas de procesos de transición de un nivel cognitivo a otro (Kuhn 1962/2002a:11). En la medida en que la psicología del conocimiento le ha permitido a Kuhn la comprensión de cambios científicos en la historia de la ciencia, no parece haber encontrado dificultad en pensar en la equivalencia entre ambas cosas.

Conviene también realizar las siguientes observaciones ampliatorias. Es verdad que los cambios revolucionarios, según Kuhn, ocurren a nivel de la comunidad, por lo tanto son histórico-comunitarios. Una revolución no la realiza un hombre, pero alguno o algunos la comienzan. Así lo entiende Kuhn cuando asume que Copérnico y Einstein realizaron (gracias a los cambios en sus esquemas cognitivos) verdaderas revoluciones científicas posibilitando un salto entre paradigmas incompatibles (Kuhn 1962/2002a:158). La revolución es progresiva, de modo que otros la deben continuar, de hecho Kuhn habla de microprocesos (Kuhn 1989/2002b:111) sugiriendo ajustes siguientes. En dichos microprocesos los individuos están implicados en instancias de modificación de conceptos previos que exigen realizaciones de cambios conceptuales a escala menor. En un largo proceso los pequeños cambios conceptuales puede que no siempre merezcan recibir el sentido pleno o evidente de revolución. Pero la colaboración en esa transición indica un pensamiento necesariamente divergente (Kuhn 1959/1996) que se verifica por la no aceptación sumisa de un paradigma común. Ignorar esto sería subestimar el número de científicos que participan en un proceso revolucionario, que cuanto más extendido esté en el tiempo más aumentará. No sólo los científicos que realizan sus aportes originales deben efectuar estos cambios conceptuales, sino todos los miembros de la comunidad que se convencen de sus propuestas.

Por supuesto que Copérnico no completó la revolución copernicana: con ese nombre se ha llamado a un itinerario científico comenzado por él y que se considera finalizado con Newton. Sin embargo, es difícil decir que Copérnico no aportó un cambio fundamental respecto de la concepción astronómica de su tiempo. La vaguedad de la noción de paradigma colabora, en este caso, positivamente a fin de no delimitarlo entre un antes y un después muy precisos. En los microprocesos de cambio puede entenderse que el paradigma sufre sucesivas modificaciones locales que lo van reajustando hasta un equilibrio más logrado. La revolución copernicana comenzó cambiando el centro geométrico del universo, necesitó luego la modificación de las leyes del movimiento: la ley de inercia, la noción de fuerza, hasta el concepto de gravitación. Ahora bien, siempre que sucedan este tipo de ajustes sucesivos debe haber algún cambio cognoscitivo importante en la consideración de los científicos. Lo dicho anteriormente, respecto de la vaguedad del límite de comienzo y fin de un paradigma, nos permite pensar tanto que la revolución científica funciona a largo plazo como en esos pequeños, pero decisivos, cambios conceptuales que la van delineando. Es aquí donde la participación 
de los científicos no se verifica como la de una aceptación masiva e irreflexiva. Dentro de la comunidad hay mentes que piensan como completar el paradigma y darle mayor coherencia. Ellos son los responsables también del cambio científico. Desentenderse de la experiencia particular y del aparato cognitivo de los sujetos concretos, que operan en medio de las revoluciones, es desconocer la mecánica interna de los cambios históricos.

\subsection{El tema de la continuidad en el cambio teórico y en la psicogénesis}

Piaget y García (2000) tomaron a su cargo la tarea de mostrar que las herramientas que proporcionan el método psicogenético y la verificación de los procesos históricos de la ciencia permiten pensar en mecanismos comunes a ambos. La comparación entre psicogénesis y la historia de la ciencia puede justificarse sólo si se muestran los mismos principios. Según Castorina, "el empleo coordinado del método psicogenético y de una reconstrucción de la historia de la ciencia dio lugar a las hipótesis -aún por verificar en diversos dominios del conocimiento- acerca de mecanismos e instrumentos comunes a la psicogénesis y la formación histórica de las ciencias [según Piaget y García]. Por ejemplo, los procesos de desestabilización de los sistemas de conocimiento y su ulterior reequilibración por medio de las abstracciones y generalizaciones, la invención de novedades y su articulación" (Castorina 1998:25).

A decir verdad Piaget y García reconocieron el parentesco con la postura kuhniana sólo parcialmente. La continuidad del desarrollo del sistema cognoscitivo desde el niño hasta el hombre de ciencia muestra, según ellos, una correspondencia con el desarrollo de la física desde Aristóteles hasta la actualidad. Esa continuidad no excluye las discontinuidades. Lo único que se mantiene en todas las etapas, uniéndolas, es el funcionamiento y no el carácter de las estructuras. Estos factores funcionales son las conocidas invariantes, en especial los aspectos que conforman la adaptación cognoscitiva, es decir, la asimilación y la acomodación, y los procesos de equilibración implicados. Es el cambio de estructuras el aspecto que evidencia la discontinuidad o salto en el proceso. Según Piaget y García estos son puntos de acuerdo con Kuhn (cfr. Piaget y García 2000:237). Lo que no acuerdan con él es que las reestructuraciones, si bien pueden concebirse como saltos, no posean una lógica interna.

Las diferencias con la epistemología de Kuhn se pueden encontrar claras en este pasaje: “Kuhn, por el contrario, no acepta que haya continuidad en la evolución científica, ni que haya mecanismos claros que permitan sustituir un paradigma por otro. Él comprueba el hecho de que un paradigma es desalojado históricamente por otro, sin que haya normas cuya aplicación explique cómo ocurre. Más aún, su concepción de paradigma torna imposible establecer criterios que permitan comparar dos de ellos entre sí para establecer la superioridad de uno sobre el otro. En ello consiste precisamente su idea de la inconmensurabilidad de los paradigmas. De aquí surge la frecuente acusación de irracionalista que le han hecho sus críticos: en la concepción de Kuhn no hay lugar para el progreso científico" (Piaget y García 2000:238).

El análisis precedente es sin duda parcial, está basado en el Kuhn de La Estructura. Es sabido que Kuhn sostuvo una idea de progreso científico, aceptó la existencia de criterios para la preferencia de un paradigma sobre otro y, fundamentalmente, modificó (o aclaró) su noción de inconmensurabilidad dando lugar a una concepción menos radical, más acotada o parcializada de la misma. También debe puntualizarse que el carácter local de la inconmensurabilidad permite pensar la continuidad de ciertos aspectos en el cambio científico (más adelante ampliaremos con más justificación esta consideración). Pero es lógico que todos estos tratamientos no aparecieran en la obra de Piaget y García, porque ésta es anterior a lo que se 
reconoce como el último Kuhn. Recién a partir de los años ochenta Kuhn pasa a reelaborar sus ideas con un matiz lingüístico y atenuando las interpretaciones más extremas de La Estructura.

\subsection{Las crisis en la ciencia como crisis cognitiva}

Según la opinión de Greiffenhagen y Sherman (2008) sería incorrecta la comparación entre los momentos de crisis de la ciencia con lo que ocurre en los conflictos cognitivos a nivel del psiquismo individual. Los autores señalan que el concepto de crisis, trabajado por Kuhn, atañe solamente a la ciencia en cuanto institución, y ése es el único sentido en que hay que tomar sus palabras. Es verdad que los momentos de crisis son instancias de replanteo a todo nivel. Es de esperar que se produzcan conmociones a nivel institucional, se vean afectados los organismos encargados de la difusión del conocimiento y cambien parámetros de investigación y de distribución de recursos. Pero esta crisis es básicamente epistemológica, es decir, está referida al cuerpo de conocimientos que ya no se puede seguir sosteniendo. $Y$ el conocimiento es un fenómeno primariamente individual que puede socializarse. Kuhn tardó en reconocer que las comunidades no son entes con experiencias cognitivas (Kuhn 1989/2002b:111), lo cual no significa que no puedan reproducir hasta cierto punto procesos individuales. Conviene aquí, además, recordar que, justamente cuando Kuhn describe el estado de la ciencia en crisis no puede dejar de citar a los actores individuales describiendo el mundo interno del científico. El nuevo paradigma suele surgir de la mente de un hombre que está sumergido "profundamente" en la crisis (Kuhn 1962/2002a:146).

El momento de crisis es una conmoción intelectual cuyas repercusiones, por qué no decirlo, son casi existenciales. Según Kuhn: “Einstein, limitado por el uso corriente a un lenguaje menos florido, escribió solamente: 'Es como si le hubieran retirado a uno el terreno que pisaba, sin ver en ninguna parte un punto firme sobre el que fuera posible construir'. Y Wolfgang Pauli, en los meses anteriores al momento en que el documento de Heisenberg sobre la mecánica matricial señalara el camino hacia una nueva teoría cuántica, escribió a un amigo: 'Por el momento, la física se encuentra otra vez terriblemente confusa. De cualquier modo, es muy difícil para mí y desearía haber sido actor de cine o algo parecido y no haber oído hablar nunca de la física" (Kuhn 1962/2002a:137-138).

Estas confesiones son de hombres de ciencia cuyos aparatos conceptuales, con los que habían venido trabajando, se encuentran afectados de tal modo que no pueden comprender lo que hasta entonces comprendían. No hay mejor modo de entender una crisis científica que tomando en cuenta la crisis en los sujetos epistémicos. El disconformismo sobre las explicaciones disponibles y la verificación de la insuficiencia de los recursos cognitivos son hechos eminentemente individuales de alcance epistémico. La crisis de la ciencia, antes que ser la de una institución, no es más que el reflejo de la crisis por la que atraviesan los científicos concretos.

Ya sea porque la revolución científica no se completa de la noche a la mañana y requiere de la intervención de varias mentes lúcidas y creadoras de nuevos esquemas de pensamiento y resolución de problemas, o por la prueba histórica de que los hombres concretos y sus aparatos conceptuales están siendo exigidos en medio de una crisis; es evidente que en los cambios científicos, según Kuhn, juegan un papel fundamental los factores psico-cognitivos. Hay, por lo tanto, suficientes elementos probatorios para sostener que un tema de interés kuhniano permanente ha sido cómo piensan los científicos. 


\section{El problema de la cientificidad de la psicología en Kuhn}

Una de las objeciones más importantes que podría recibir el planteo propuesto consistiría en la imposibilidad, desde la perspectiva de Kuhn, de darle crédito científico a la psicología. En tal caso, sería dudoso que él atendiera demasiado a lo que la disciplina en cuestión podría ofrecerle. Tal como Kuhn formula su epistemología histórica, la psicología no tendría derechos suficientes para ser considerada una ciencia. Teniendo en cuenta el esquema de Kuhn, en relación con el desarrollo de la ciencia, parecería lógico ubicar a la psicología, al igual que a las demás ciencias sociales, dentro de lo que se ha llamado período de pre-ciencia o pre-paradigmático. En esta ubicación la psicología no ofrecería la confiabilidad suficiente para ser considerada una ciencia $y$, por lo tanto, tampoco podría significar demasiado que Kuhn, en su descripción del resto de las ciencias, hiciera referencia a algunas de las teorías que surgen de la psicología. En otras palabras, el programa de la epistemología de Kuhn que incluye: el análisis de las ciencias desde su génesis, el establecimiento de fértiles tradiciones de investigación, los factores de producción del conocimiento científico, el establecimiento de marcos de comprensión teóricos y sus transformaciones progresivas; no podría entregarse seriamente a una disciplina no científica como la psicología. El recurso a la psicología, en consecuencia, no tendría en Kuhn más que un valor relativo y hasta prescindible. Este no es un tema de sencilla resolución y exige una respuesta compleja.

En primer lugar, las expresiones pre-ciencia o pre-paradigma, referidas al período anterior al de la ciencia madura, pueden resultar desorientadoras. Una interpretación posible es que en este período no existe algo que se pueda llamar realmente ciencia. Aunque Kuhn es aquí tan ambiguo como en otros casos al decir que, durante este período, puede que haya científicos sin ciencia (Kuhn 1962/2002a:37). Aun aceptando que no hubiese ciencia es de notar que el capítulo II de La Estructura de las Revoluciones Científicas no es Ilamado: El período pre-científico sino, sugerentemente: Hacia la ciencia normal. Entonces ¿puede haber práctica científica dentro de algún tipo de ciencia no-normal? Hay que tener en cuenta, también, que el período hacia la ciencia madura no carece absolutamente de paradigmas, más bien es de esperar que haya muchos que se encuentren en disputa. Cuando Kuhn decide corregir algunas de sus primeras imprecisiones entiende (justamente, según admite, por ciertas exigencias llegadas desde las ciencias sociales) que el pasaje a la ciencia madura no se da necesariamente con la adquisición de un paradigma como si antes no hubiese existido ninguno.

Como señala en la Posdata de 1969: “La naturaleza de esa transición a la madurez merece un análisis más completo del que ha recibido en este libro, particularmente de aquellos interesados en el avance de las ciencias sociales contemporáneas. Con ese fin puede ser útil indicar que la transición no tiene que estar asociada (ahora creo que no debe estarlo) con la primera adquisición de un paradigma. Los miembros de todas las comunidades científicas, incluso de las escuelas del período 'preparadigma' comparten las clases de elementos que, colectivamente, he llamado un 'paradigma'. Lo que cambia con la transición a la madurez no es la presencia de un paradigma, sino, antes bien, su naturaleza. Sólo después del cambio es posible una investigación normal de la solución de enigmas" (Kuhn 1969/2002a:274-275).

Es decir, la diferencia entre el período pre y post paradigma es la reducción de un número de escuelas a una sola que toma el mando en la solución de enigmas. No hay en esto ninguna indicación de que las prácticas de las escuelas anteriores a la aparición de la ciencia normal deban ser necesariamente menoscabadas. Entonces ¿en qué sentido se puede hablar de que antes de la ciencia normal no hay ciencia? 
Hay aquí otra arista de la cuestión que conviene mencionar. Al hablar de investigaciones cargadas de inmadurez, los ejemplos adecuados de Kuhn son válidos para las ciencias naturales. La inmadurez propia de la prehistoria de una ciencia natural mostraría su desorganización en cuanto a la interpretación de los datos y la falta de un principio ordenador de los fenómenos que se yuxtaponen conduce necesariamente a una sensación de agobio intelectual. Según Kuhn: "A falta de un paradigma o de algún candidato a paradigma, todos los hechos que pudieran ser pertinentes para el desarrollo de una ciencia dada tienen probabilidades de parecer igualmente importantes (...) cualquiera que examine, por ejemplo, los escritos enciclopédicos de Plinio o las historias naturales baconianas del siglo XVII, descubrirá que el producto es un marasmo" (Kuhn 1962/2002a:41).

No puede decirse que suceda lo mismo en el campo de las ciencias sociales, al menos con muchas de las teorías que se proponen. En ciencias sociales los presupuestos teóricos suelen ser tan firmes como en las ciencias naturales, la interpretación de los fenómenos responde habitualmente a una noción base que esclarece las razones de los hechos y, por último, la comunidad de los especialistas que adhieren a esa escuela o corriente de pensamiento tienen actividades similares a los científicos que Kuhn describe en el período de ciencia normal. Lo que evidentemente no significa que funcionen con exclusividad ya que habrá otros grupos que utilicen otros principios y expliquen los mismos fenómenos de otra manera. Esta característica de las ciencias sociales las coloca, en el momento de la investigación científica, antes del establecimiento de un paradigma único. Lo que no puede decirse es que, en ellas, todo suceda con la misma sistematicidad y homogeneidad como en las ciencias naturales en su período normal.

Lo cierto es que las prácticas no normalizadas de la psicología no carecen de atención de parte del Kuhn epistemólogo. En sus escritos nunca se ha referido a la psicología de un modo peyorativo, sino todo lo contrario. Tampoco lo ha hecho con el conjunto de las ciencias sociales. Es más, Kuhn no cuestiona la expresión "ciencias sociales" o "ciencias humanas", las cuales utiliza abiertamente. En un artículo expuesto en 1989 y publicado por primera vez en 1991 titulado Las ciencias naturales y las humanas, aborda este tema tratando de establecer diferencias entre ambos tipos de ciencias. Lo que surge de este texto es una clara demostración de su indefinición respecto de la posibilidad de que las ciencias humanas alcancen a funcionar o no algún día según el modelo de las naturales. En lo fundamental Kuhn sostiene ahí que las ciencias humanas, al ser hermenéuticas, pueden ver dificultado su acceso a la investigación normal que permita la resolución de enigmas (Kuhn 1991/2002b:264), tal como sucede en las ciencias naturales. Esto significaría, en apariencia, una inmadurez de las ciencias humanas imposible de salvar. Pero al mismo tiempo, Kuhn admite su duda respecto de que tal dificultad no pueda eliminarse nunca. Expresamente menciona a la economía y a la psicología como ciencias que podrían estar en transición hacia este estado de resolución normalizada de enigmas. Tal vez Kuhn nunca haya podido abandonar una especie de prejuicio respecto de la cientificidad de las ciencias sociales, propio de los que provienen de las ciencias físicas y naturales. Por eso mantuvo hasta el final su ideal de normalización de una disciplina para poder encuadrarla dentro de su esquema general del desarrollo científico. Aunque, evidentemente, este ideal se ve dificultado en las ciencias humanas y él es consciente de ello.

La posible consideración de la falta de cientificidad de la psicología en Kuhn y, por lo tanto, de la invalidación del uso riguroso de esa ciencia en su obra, es algo que hay que pensar aún hoy. Tal vez se trate de algo que él no terminó de aclararse, pero que nosotros estamos en condiciones de hacerlo. En principio, la potencialidad de las ciencias sociales o humanas supera cualquier análisis demasiado estrecho en el que no se las pueda considerar ciencias. Un criterio demarcatorio con las ciencias naturales, como el que Kuhn 
intenta, no significa desvalorización de su peso epistémico sino reconocimiento de una naturaleza diferente. Pero ¿puede haber ciencias que no sean similares en su práctica a las naturales? Un poco de luz podría echarse sobre el tema si traemos a colación el concepto de verdad en Kuhn. Sin entrar en detalles exhaustivos puede decirse que la concepción que maneja Kuhn no remite a una verdad última y definitiva. La inconmensurabilidad entre paradigmas hace que ninguno pueda proclamarse como más verdadero. En las ciencias naturales existe un acuerdo transitorio de la comunidad respecto del paradigma a defender, pero sabemos que este acuerdo puede modificarse. Al no haber paradigma absoluto no puede definirse si el paradigma anterior o el nuevo muestran un acercamiento a la verdad o el en sí de las cosas. Lo mismo sucede en las ciencias sociales, con la diferencia que en lugar de mostrar, en un despliegue histórico la imposibilidad de un paradigma definitivo, evidencian la misma situación en una coexistencia temporal de teorías no asimilables. ¿Por qué sería más confiable la ciencia natural, con muchos paradigmas que se suceden, que una ciencia con paradigmas que coexisten? ¿Por qué deberían ser preferibles los saberes que se contradicen en secuencias diacrónicas? ¿Qué ventaja hay en ello por sobre los saberes que reconocen oposiciones insalvables en forma sincrónica? Podría argumentarse que Kuhn exige la presentación de la ciencia normal para hablar seriamente de conocimiento científico. Pero ¿qué nos haría pensar que Kuhn no tomara con valor científico lo que los distintos paradigmas psicológicos le ofrecieran?

El planteo de la cientificidad de la psicología en la obra de Kuhn puede representar ciertamente un problema. Pero este problema está originado por los límites de la epistemología kuhniana, contra los que él mismo se vio enfrentado y más de una vez debió revisar. Es probable que Kuhn abrigara la esperanza de que la psicología alcanzara un modelo unificador, como algunos suponen puede pretenderlo lograr el cognitivismo. Pero tal unificación no sólo no es deseable sino que desfiguraría el campo científico propio de la psicología. En el estado actual de la situación hay que admitir que la psicología, al igual que las demás ciencias sociales, es multiparadigmática por esencia. No existe ninguna necesidad ni urgencia de homogeneizar, en una forma explicativa general, cuestiones tan diversas que componen estos campos de investigación.

Por lo demás, y en cuanto al uso que hace Kuhn de la psicología del conocimiento en su obra, no evidencia ningún descrédito hacia ninguna de las teorías a las que acude de un modo explícito o implícito. Las investigaciones de la Gestalt, o de Piaget, como las de la psicología cognitiva en general, son tomadas por Kuhn con absoluta seriedad y como informes de alta relevancia para entender algo del comportamiento del campo científico. La utilización alternada de estas distintas teorías manifiesta su falta de adopción de un criterio único y pone a las claras el carácter multiparadigmático de la psicología, pero de ninguna manera el menosprecio de Kuhn respecto de los estudios de ese campo.

\section{Conclusión}

La psicología intenta explicar los procesos cognitivos del ser humano. Entre ellos, con particular interés, nos hemos detenido en los factores que inciden en los cambios científicos. Según Kuhn, en las crisis y cambios científicos juegan un papel fundamental los procesos psico-cognitivos. El interés manifestado por Kuhn a lo largo de su obra por la psicología del conocimiento es constante. Desde aproximaciones transitorias, como a la Gestalt o el asociacionismo, hasta uniones más prolongadas como con la psicología cognitiva o la psicología genética, Kuhn no ha dejado a lo largo de toda su obra espacio para la duda acerca de su interés en las teorías psicológicas. Esto nos lleva a ubicar a Kuhn dentro de los investigadores que han desarrollado desde su marco epistémico una verdadera psicología de la investigación. 
Por otro lado, las investigaciones, que Kuhn llevó adelante en el campo de la historia de la ciencia, tienen vigencia para ayudarnos a pensar el estatuto epistémico de la psicología y su peculiar naturaleza científica. Pero además son un claro ejemplo de la exigencia interdisciplinaria propia del campo de investigación psicológica. Esta exigencia es acorde a la complejidad del comportamiento humano, objeto de estudio compartido por las ciencias sociales. Lejos de denostar a la psicología como pseudocientífica, Kuhn señala que lo propio de las Ciencias Sociales es el carácter multiparadigmático y no las convoca como otros científicos a abandonar su carácter sui generis en pos de un ropaje positivista. Por el contrario, se trata de mantener y sostener la cientificidad de la psicología desde su riqueza pluriparadigmática.

\section{Notas}

(1) Este artículo es parte del Proyecto P 404: Estrategias didácticas en el desarrollo de la sensibilidad moral en la educación. Facultad de Psicología de la UBA.

(2) Thomas S. Kuhn. The Quest for Physical Theory: Problems in the Methodology of Scientific Research, 3-30III-1951, Thomas S. Kuhn Papers [MC 240], Institute Archives and Special Collections, Massachusetts Institute of Technology, Cambridge, Mass., caja 33, carpeta 11. La referencia y los comentarios que se desarrollan sobre este tema en el presente trabajo han sido tomados de Mayoral de Lucas (2004).

\section{Bibliografía}

Baltas, A., Gavroglu, K. y Kindi, V. 2002. Una conversación con Thomas Kuhn. En: T. Kuhn. 2002b. El camino desde la estructura. Barcelona: Paidós, pp. 299-373.

Carey, S. 2004. Bootstrapping \& the origin of concepts. Daedalus 133: 59-68.

Castorina, J. A. 1998. Los problemas conceptuales del constructivismo y sus relaciones con la educación. En: M. Carretero, J. Castorina y R. Baquero (eds). Debates constructivistas. Buenos Aires: Aique, pp. 21-45.

Greiffenhagen, C. y Sherman, W. 2008. Kuhn and conceptual change: On the analogy between conceptual changes in science and children. Science \& Education 17(1): 1-26.

Kuhn, T. S. (1991/2002b). Las ciencias naturales y las humanas. En: T. Kuhn. El camino desde la estructura. Barcelona: Paidós, pp. 257-265.

Kuhn, T. S. (1989/2002b). Mundos posibles en la historia de la ciencia (artículo presentado en el 65 Symposium Nobel 1986). En: T. Kuhn. El camino desde la estructura. Barcelona: Paidós, pp. 77-112.

Kuhn, T. S. 1976/2002b. Cambio de teoría como cambio de estructura: comentarios sobre el formalismo de Sneed. En: T. Kuhn. El camino desde la estructura. Barcelona: Paidós, pp. 211-232.

Kuhn, T. S. 1974/1978. Segundos pensamientos sobre paradigmas. Madrid: Tecnos.

Kuhn, T. S. 1971/1996. Los conceptos de causa en el desarrollo de la física. En: T. Kuhn. La tensión esencial. México: FCE, pp. 46-55.

Kuhn, T. S. (1969/2002a). Posdata. En: T. Kuhn. La estructura de las revoluciones científicas. Bs. As: FCE, 268319. 
Kuhn, T. S. (1962/2002a). La estructura de las revoluciones cientificas. Bs. As: FCE.

Kuhn, T. S. (1959/1996). La tensión esencial: tradición e innovación en la investigación científica. En: T. Kuhn. La tensión esencial. México: FCE, pp. 248-262.

Kuhn, T. S. 1957. La revolución copernicana. Madrid: Orbis.

Mayoral de Lucas, J. V. 2004. Thomas S. Kuhn y el lenguaje científico, 1949-1951: Los argumentos filosóficos de las Conferencias Lowell. En: Actas del IV Congreso de la Sociedad de Lógica, Metodología y Filosofía de la Ciencia. Valladolid, 3-6 de noviembre de 2004. http://bit.ly/dyUlKW

Nersessian, N. J. 2003. Kuhn, conceptual change, and cognitive science. En: T. Nickles (ed). Thomas Kuhn. Cambridge: Cambridge University Press, pp. 178-211.

Rosch, E. 1999. Reclaiming concepts. The Journal of Consciousness Studies 6(11/12): 61-77.

Piaget, J. y García, R. 2000. Psicogénesis e historia de la ciencia. México: Siglo XXI.

Recibido el 22 Mar 2010

Aceptado el 28 Jun 2010 